\title{
Bei chronischer Sinusitis unters Messer
}

\author{
Eine chronische Sinusitis bleibt oft monatelang, kommt immer wieder und schränkt \\ dabei viele Betroffene stark ein. Eine multizentrische Kohortenstudie zeigte nun, dass \\ die minimal-invasive endoskopische Sinuschirurgie die Lebensqualität auch langfristig \\ signifikant verbessert.
}

Datienten mit chronischer Rhinosinusitis leiden besonders stark unter ihrer Erkrankung: Sie haben eine schlechtere Lebensqualität als Patienten mit Stauungsinsuffizienz, Angina, COPD oder Rückenschmerzen. Standardmedikation wie abschwellende Nasentropfen, Schmerzmittel, Wärmepackungen, Kochsalz-Nasensprays und Inhalationen helfen oft nicht. Schließlich werden oft chirurgische Eingriffe durchgeführt mit dem Ziel, ein gewisses Ausmaß an Erleichterung zu verschaffen.
In einer umfangreichen prospektiven Studie wurde nun untersucht, bei wie vielen Patienten mit chronischer Rhinosinusitis sich die Lebensqualität durch den endoskopischen Eingriff signifikant besserte. Durch validierte, Sinusitis-spezifische diagnostische Messungen erhofften sie sich zudem Auskunft darüber zu erhalten, welche Patienten besonders von der Operation profitieren. So ermittelten sie präoperative Patientendaten, CTScan, endoskopischen Score, sowie präund postoperative Quality-of-Life

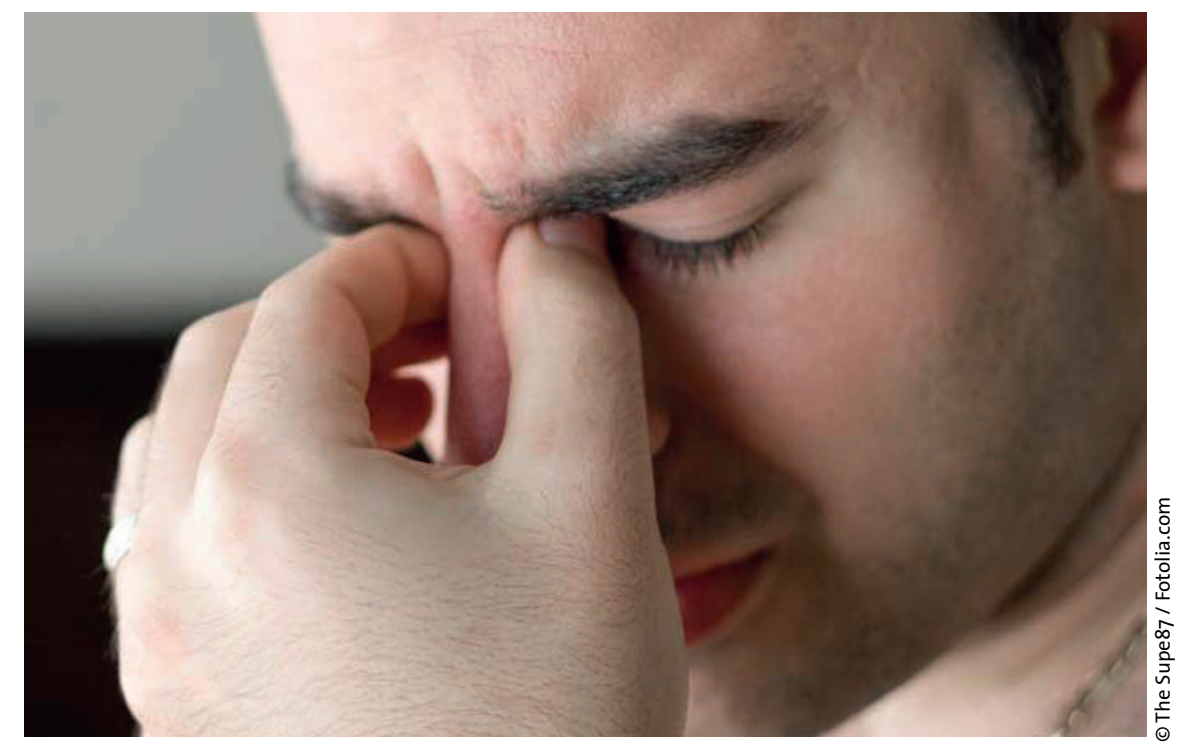

(QOL)-Daten. Insgesamt wurden 302 Erwachsene mit chronischer Rhinosinusitis an drei verschiedenen Zentren zwischen Juli 2004 und Dezember 2008 überprüft und postoperativ über durchschnittlich 17,4 Monate nachverfolgt.

Zum Studienende zeigten gut drei Viertel der Teilnehmer nach dem Eingriff eine deutlich höhere Lebensqualität, weniger körperliche Leiden und eine bessere soziale Funktion. So verbesserten sich die Patienten um durchschnittlich 15,8\% $(18,9$ Punkte) im Rhinosinusitis-Disability-Index (RSDI) und zu 21,2\% (21,2 Punkte) beim Chronische-Sinusitis-Survey (CSS). Von den Patienten mit schlechtem präoperativem QOL erfuhren $71,7 \%$ eine klinisch signifikante Verbesserung im RSDI und 76,1\% im CSS. Bei einer Erstoperation war die Wahrscheinlichkeit für RSDI- oder CSS-Verbesserungen dabei rund doppelt so hoch wie bei einem Revisionseingriff.

Fazit: Ein operativer Eingriff bei chronischer Rhinosinusitis schlägt sich unmittelbar in der Lebensqualität des Patienten nieder.

bsk

Smith TL et al. Determinants of outcomes of sinus surgery: a multi-institutional prospective cohort study. Otolaryngol Head Neck Surg 2010;142:55-63

\section{Metastasendilemma - ein Fall für Sorafenib?}

\section{Beim differenzierten Schilddrüsenkarzinom mit Radiojod-refraktärer Metastasierung gibt es kaum aussichtsreiche Therapieoptionen. Angesichts der Beteiligung von Tyrosinkinasen an den Signalwegen dieser Tumoren sind Multi-Kinase-Hemmer einen Versuch wert.}

Zis. wei Studien mit Sorafenib wurden bereits publiziert. Sie umfassten nicht nur Patienten mit differenziertem Schilddrüsenkarzinom, sondern auch solche mit anaplastischem und in einer Studie außerdem mit medullärem Karzinom. Bei 23 bzw. $11 \%$ der Patienten fand sich ein partielles Ansprechen; das mediane progressionsfreie Überleben betrug 79 bzw. 4,5-16 Wochen. Eine separate Analyse für Patienten mit Knochenmetastasen gab es aber nicht. Da diese Metastasen kaum auf Radiojod ansprechen, wäre ihr Verhalten unter Multi-Kinase-Hemmung von besonderem Interesse.

Eine weitere Studie widmete sich deshalb vor allem den Effekten von Sorafenib (400 mg zweimal täglich) auf die Rein- 